\title{
Physical activity among adults with diabetes mellitus in Rwanda
}

\author{
A.M. KABANDA AND J.S. PHILLIPS \\ Department of Physiotherapy, University of the Western Cape, P. Bag X17, Bellville 7535. E- \\ Mail: jphillips@uwc.ac.za
}

(Received 20 May 2010; Revision Accepted 23 February 2011)

\begin{abstract}
Diabetes mellitus is one of the most common non-communicable diseases, and is the fifth leading cause of death in most developing countries. Regular physical activity is strongly recommended for individuals with diabetes for its beneficial effects in the improvement of blood glucose control and insulin sensitivity, prevention and reduction of morbidities and complications, as well as for its cardiovascular benefits. Using a cross-sectional design, this study examined the demographic, social and health-related factors associated with physical activity participation among adults with diabetes mellitus in Kigali, Rwanda. One hundred-and-fifty six (156) adults with diabetes mellitus participated in the study. More than a third $(39 \%)$ of the participants was categorized as inactive. Active participants were significantly younger than those categorized as inactive. Age, marital status, level of education and self-efficacy for diabetes were all significantly associated with levels of physical activity. Common barriers to participation in physical activity were cited as poor health status, lack of motivation and lack of awareness about the importance of physical activity. It is thus clear that efforts should be made to educate adults with diabetes mellitus about the benefits of integrating regular physical activity in their daily routine.
\end{abstract}

Key words: Diabetes mellitus, physical activity, adults, Rwanda.

\section{Introduction}

Non-communicable diseases, such as diabetes mellitus, cardiovascular disease, stroke, cancer and chronic respiratory diseases are currently the leading cause of mortality in the world, representing $60 \%$ of all deaths (World Health Organization, 2008). Diabetes mellitus, long considered of minor significance to world health, is now taking its place as one of the main threats to human health in the $21^{\text {st }}$ century (Zimmet, Alberti \& Shaw, 2001). The International Diabetes Federation (IDF) estimates that there were 189 million people with diabetes in 2003 and predicts an increase to 324 million in 2025 (IDF, 2005). Similarly, the World Health Organization (WHO) in 2006 reported that by the year 2025, there will be an almost $170 \%$ increase in the number of diabetics in developing countries, while the increase in developed countries will be about $42 \%$. Furthermore, the IDF and WHO (2006a) reported in a Joint initiative of IDF Africa and WHO-AFRO that the expected majority of the increase in numbers of people with diabetes will be predominantly in the 45-64 year age group 
particularly in developing countries. This is of particular economic importance, as people will be affected in the most productive period of their lives.

In order to reduce the impact of diabetes worldwide, the WHO and IDF in 2006 launched the Diabetes Action Now programme. The programme focuses on low and middle-income communities, particularly in developing countries and its purpose is to stimulate and support the adoption of effective measures for the surveillance, prevention and control of diabetes. In addition, the key aim of the programme is to achieve a substantial increase in the global awareness about diabetes and its complications (WHO, 2006a). Effective education may thus produce changes in knowledge and understanding and may even result in a change in behaviour or lifestyle.

Physical activity plays a significant role in both the prevention and management of diabetes mellitus (Kriska et al., 2003). Physical activity has the potential to provide several benefits for the diabetic individual which may include: possible improvement in blood glucose control, improvement of insulin sensitivity thus requiring less medication, reduction in body fat, cardiovascular benefits and stress reduction. Similarly, regular physical activity is often recommended for individuals with diabetes in view of its beneficial effects on the metabolic risk factors often associated with diabetes complications (Nakawatase et al., 2007). All levels of physical activity, including leisure activities, recreational sports, and competitive professional performance can be performed by people with diabetes who do not have complications and have good glucose control (American Diabetes Association, 2003). However, despite these and other benefits, many individuals with diabetes do not include regular physical activity as a part of their diabetes care (Chau \& Edelman, 2001). Despite the fact that the importance of exercise and physical activity is probably stressed more to diabetics compared with non-diabetics, diabetic individuals are less likely to exercise than non-diabetic individuals (Nakawatase et al., 2007). Individuals with chronic illness often avoid exercise for fear of making their condition worse or overexerting themselves, yet these patients are the ones that stand to benefit most from a regular exercise programme (Kolt \& Snyder-Mackler, 2003).

There is thus a need to determine the levels of participation in physical activity among adults with diabetes mellitus in developing countries and understand their association with demographic, social and health-related factors, in view of the benefits of engagement in physical activity by diabetics. Therefore, the aim of this study was to establish the levels of participation in physical activity and the factors associated with it, among adults with diabetes mellitus in Kigali, Rwanda. 


\section{Methods}

The Rwandan Diabetic Association (RDA) specialized clinic, situated in Kigali, Rwanda, was chosen as the research setting as it receives most of the diabetic patients from rural and urban areas of Rwanda. The clinic that was created after the 1994 genocide provides 24-hour care for people with diabetes mellitus and was designed to prevent, promote treat and rehabilitate diabetic patients. Approximately 390 adults aged 18 years and older attend the RDA clinic on a monthly basis. A systematic random sampling technique was used to select participants for the study. Every $2^{\text {nd }}$ patient who came for treatment or follow-up care at the clinic in a specified time was approached to participate in the study. A final number of 156 adults with diabetes mellitus finally agreed to participate in the study.

Permission and ethical clearance for the study was obtained from the Senate Research Grants and Study Leave Committee at the University of the Western Cape, South Africa. Permission was also obtained from the Ministry of Health in Rwanda and the President of the Rwandan Diabetic Association.

The study utilized a quantitative design. Three questionnaires were administered to adults with diabetes at the RDA specialized clinic. The first questionnaire was a self-developed questionnaire requesting for demographic details such as gender, age, height, weight, highest educational level and marital status. The type of diabetes mellitus was also noted in this section. The researchers consulted the participants' medical records to ensure the validity of the diagnosis. One question assessed the participants' perception regarding their health status and was measured by the item "How would you rate your overall health at the present time?" This item had been used in previous research and its validity has been supported by Idler and Benyamini (1997) that identified selfreported health status as a significant predictor of mortality among adults.

The second questionnaire measured participants' self-efficacy concerning diabetes. It aims at identifying how confident participants are in dealing with diabetes mellitus in their activities of daily living. This scale has been used in previous research and has an internal consistency reliability of 0.83 . Physical activity levels were assessed by the International Physical Activity Questionnaire (IPAQ). This consists of 15 questions which assess the physical activities related to work, transport and leisure-time. The IPAQ was first used in Geneva in 1998 and has undergone extensive reliability and validity testing across 12 countries. Craig et al. (2003) concluded that the IPAQ has acceptable measurement properties for use in many settings and in different languages. Lastly the participants were asked if they were prevented from being physically active, and if yes, to list possible barriers to physical activity participation. 
The questionnaire was translated from English to Kinyarwanda by a professional translator. Kinyarwanda is the common language spoken by the majority of Rwandan people. To ensure the validity of the instrument, the Kinyarwanda questionnaire was then back-translated into English by an independent translator. The second version was shown to be similar to the original English version of the questionnaire. To further ensure validity, the questionnaire was given to the medical personnel of the RDA clinic for their views and suggestions with regard to the clarity of the questionnaire and the objectives to be met.

\section{Data analysis}

Descriptive statistics were calculated for demographic variables and healthrelated factors, such as BMI, perceived health status, self-efficacy concerning diabetes and physical activity levels. Cross-tabulations were used to determine the association between socio-demographic, health-related factors and physical activity levels. Chi-square analyses were undertaken to test for any significant associations. Independent samples t-test using physical activity as a categorical variable were also computed.

\section{Results}

The mean age of the participants was 51.7 (sedentary) and 45.7 (active). Participants' responses to the IPAQ were summed and the total score was calculated as MET-minutes per week for each participant. Three levels of physical activity, low, moderate and high were obtained taking into consideration the MET-minutes/week values obtained: Low (0 - 599 METminutes/week), moderate (600 - 2999 MET-minutes/week) and high (over 3000 MET-minutes/week) (Craig et al., 2003). The IPAQ assesses different types of physical activity, namely work-related, transportation-related, domestic and yard-related and leisure time physical activity. The lowest mean METminutes/week was for the study sample was obtained during leisure-time activities (233.37) as illustrated in Table 1. Moderate levels of activity were scored by $31.4 \%$ of participants during activity related to transportation.

Physical activity levels were further dichotomized into active versus inactive or sedentary. Participants who accumulated less than 599 MET-minutes/week in total, were classified as inactive or sedentary, and those who accumulated 600 and more MET-minutes/week were classified as active (Craig et al., 2003).

More than a third $(39.7 \%, \mathrm{n}=62)$ of the participants was categorized as inactive or sedentary. Participants categorized as active were significantly younger (45.7 years) than those categorized as inactive $(51.7$ years $)(p<0.05)$. The association between socio-demographic, health-related factors and physical activity participation are summarized in Table 2. A significantly higher prevalence of 
participants that were married $(52.1 \%)$ was categorized as physically active compared to those that were single (23.4\%) and separated (7.5\%).

Table 1: Levels of physical activity in different categories $(\mathrm{n}=156)$

\begin{tabular}{lllc} 
PA categories & $\mathrm{n}$ & $\%$ & Mean MET-minutes/week \\
\hline Work related: & & & $\mathbf{6 8 5 . 7 8}$ \\
Low & 123 & 78.8 & \\
Moderate & 12 & 12.8 & \\
High & 21 & 8.3 & $\mathbf{6 0 2 . 1 2}$ \\
Transport-related: & & & \\
Low & 107 & 68.6 & \\
Moderate & 38 & 31.4 & $\mathbf{5 7 5 . 3 8}$ \\
High & 11 & 7.1 & \\
Domestic and yard: & & & \\
Low & 126 & 80.8 & $\mathbf{2 2 3 . 3 7}$ \\
Moderate & 11 & 14.1 & \\
High & 19 & 5.1 & \\
Leisure-time: & & & \\
Low & 138 & 88.5 & \\
Moderate & 14 & 10.9 & \\
High & 4 & 0.6 & \\
\hline
\end{tabular}

A significantly higher prevalence of participants having a secondary school level of education were also found to be active $(45.7 \%)$ when compared to those who have reached the tertiary level of education $(4.3 \%)$. Self-efficacy regarding diabetes was also significantly associated with levels of physical activity, i.e. the majority $(87.2 \%)$ who had good self-efficacy regarding diabetes was categorized as active.

The majority of participants $(79.5 \%)$ indicated that they encountered barriers to physical activity participation. These participants were also significantly more likely to be classified as inactive $(\mathrm{p}<0.05)$. The three most likely barriers to physical activity were reported as poor health status (35.9\%), lack of motivation $(28.8 \%)$ and the lack of awareness of the importance of physical activity $(25 \%)$ as indicated in Figure 1.

\section{Discussion}

Several studies have highlighted that physical activity should be considered the cornerstone in the management of diabetes, however, it remains by far the most underused (Stys \& Kulkarni, 2007; Colberg, 2008). The findings of the current study emphasized this notion as more than one third (39.7\%) of the participating adults with diabetes mellitus were considered inactive or sedentary. 
Table 2: Factors associated with participation in physical activity $(\mathrm{n}=156)$

\begin{tabular}{lll}
\hline Variable & Sedentary $(n=62)$ & Active $(n=94)$
\end{tabular}

\begin{tabular}{lllll}
\hline & Frequency & Percentage & Frequency & Percentage \\
\hline Gender & 30 & 48.4 & 43 & \\
Male & 32 & 51.6 & 51 & 45.7 \\
Female & & & & 54.3 \\
Marital Status* & 5 & 8.1 & 22 & \\
Single & 42 & 67.7 & 49 & 23.4 \\
Married & 1 & 1.6 & 7 & 52.1 \\
Separated & 14 & 22.6 & 16 & 7.5 \\
Widowed & & & & 17.0
\end{tabular}

\section{Education Levels*}

Never went to school 16

Primary school 23

Secondary school (1-6) 9

Tertiary education $\quad 14$

25.8

$6 \quad 6.4$

37.1

$41 \quad 43.6$

14.5

$43 \quad 45.7$

22.6

4.3

\section{BMI}

Underweight

Normal weight

Overweight

Obese

\section{5}

28

16

13
8.1

45.2

25.8

20.9

4

\section{Perceived health status}

Poor

Fair

Good

Very good

Self-efficacy for diabetes*

Poor self-efficacy $\quad 18$

* Significant $(\mathrm{p}<0.05)$

This is similar to the findings of studies reported elsewhere in developed countries, in which the prevalence of inactivity ranged from $37.2 \%$ (Deshpante, Lovegreen, Baker \& Brownson, 2005) to 44\% (Morrato et al., 2007). Kolt and Snyder-Mackler (2003) postulated that patients with chronic diseases in general and diabetes mellitus in particular often avoid physical activity for fear of making their condition worse, or precipitate a hypoglycemic event. In the present study however, the majority of the participants had good self-efficacy for diabetes and therefore are fairly confident in dealing with the various aspects of their everyday life with regards to the disease. 


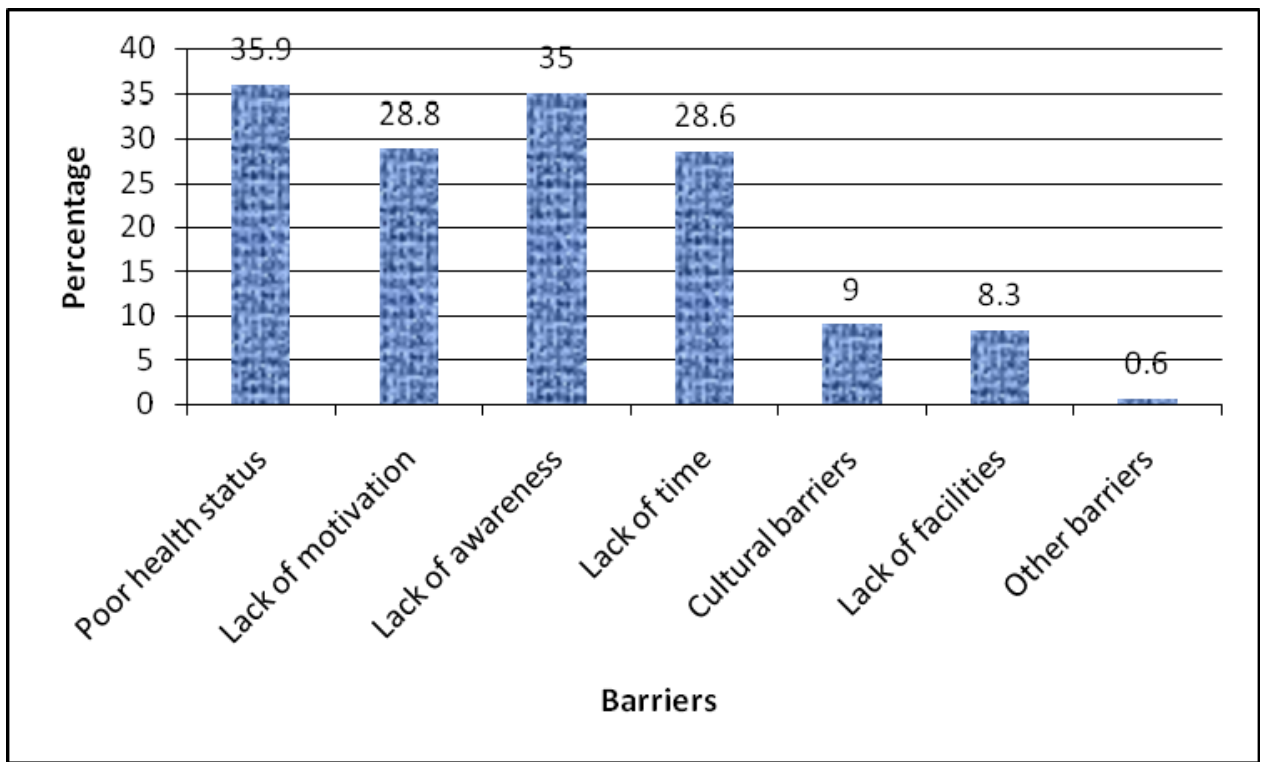

Figure 1: Barriers to participation in physical activity.

The theory of self-efficacy proposes that patients' confidence in their ability to adopt healthy lifestyles influences which behaviours they will engage in (Sarkar, Fisher \& Schillinger, 2006). It is thus clear that a good self-efficacy is not enough if not examined in conjunction with other factors such as possible barriers that adults may face with regards to physical activity participation.

Most of the participants in the current study reported low levels of physical activity during leisure-time, while moderate levels of physical activity were found in respect of transportation. The World Health Organization (2006b) reported that in poor and middle-income countries people walk more for transportation than in industrialized countries, and this has led to increased attention to walking and the need to improve the quality of this activity. A considerable number of the Rwandans, like in many other African populations, face multiple challenges caused by poverty. One of these challenges is the difficulty to afford the cost of public transportation. Although this constitutes a burden, especially for individuals with diabetes who may sometimes have to walk long distances, this may be viewed as beneficial in increasing their physical activity levels, not as a result of conscious effort, but lack of choice due to poverty. On the other hand, leisure-time physical activity could be perceived by participants as a luxury which can only be afforded by high-income individuals. This notion illustrates the need and importance of education and specific guidance of individuals with diabetes on the various types and intensity of physical activities which could be included in their daily lives. 
The factors that were shown to be significantly associated with physical activity participation in the study were age $(\mathrm{r}=0.05)$, marital status $(\mathrm{r}=0.05)$, educational levels $(\mathrm{r}=0.05)$ and self-efficacy regarding diabetes $(\mathrm{r}=0.05)$. Participants regarded as physically active were significantly younger than those considered as physically inactive in the current study. This could however be explained by the fact that even in the general population physical activity significantly decreases with age. Researchers have also indicated that inactivity is commonly seen in older patients with diabetes, and this was often associated with lack of selfefficacy, poor health status and feelings of fatigue (Thomas, Alder \& Leese, 2005).

\section{Conclusion}

The present study has shown that participants who have encountered barriers to participation in physical activity were more likely to be inactive. Tumusiime and Frantz (2006) highlighted that a person's perceived barriers to physical activity are an important determinant of how active he or she becomes. Lack of motivation and poor health status appear to be among the most common barriers to participation in physical activity faced by adults with diabetes. This finding highlights the great need for continuous support and encouragement for the integration of physical activity into adults' daily routine. Furthermore, the role of education in promoting physical activity and equipping individuals with diabetes with skills and knowledge for better self-care and adherence to management is implicated.

\section{References}

American Diabetes Association. (2003). Physical activity/exercise and diabetes mellitus. Diabetes Care, 26, 73-77.

Chau, D. \& Edelman, S.V. (2001). Clinical management of diabetes in the elderly. Clinical Diabetes, 19, 172-175.

Colberg, S.R. (2008). Encouraging patients to be physically active: What busy practitioners need to know (Practical pointers). Diabetes Care, 26(3), 123.

Craig, C.L., Marshall, A.L., Sjostrom, M., Bauman, A.E., Booth, M.L., Ainsworth, B.E., Pratt, M., Ekelund, U., Ynger, A., Sallis, J.F. \& Oja, P. (2003). International physical activity questionnaire: 12-country reliability and validity. Medicine and Science in Sports and Exercise, 35(8), 1381-1395.

Deshpante, A.D., Lovegreen, S.L., Baker, E.A. \& Brownson, R.C. (2005). Environmental correlates of physical activity among individuals with diabetes in the rural Midwest. Diabetes Care, 28, 1012-1018.

International Diabetes Federation (IDF) (2005). Prevalence all diabetes. Available at: http://www.eatlas.idf.org/prevalence/all_diabetes/html. Retrieved April 24, 2007. 
Idler, E.I. \& Benyamini, Y. (1997). Self-rated health and mortality: A review of twenty-seven community studies. Journal of Health and Social Behavior, 38, 21-37.

Kolt, G.S. \& Snyder-Mackler, J. (2003). Physical Therapies in Sport and Exercise. Champaign, IL: Human Kinetics.

Kriska, A.M., Saremi, A., Hanson, R.L., Bennet, P.H., Kobes, S., Williams, D.E. \& Knowler, W.C. (2003). Physical activity, obesity and the incidence of type 2 diabetes in a high risk population. American Journal of Epidemiology, 158, 669-675.

Morrato, E.H., Hill, J.O., Wyatt, H.R., Ghushchyan, V. \& Sullivan, P.W. (2007). Physical activity in U.S. adults with diabetes and at risk-risk for developing diabetes. Diabetes Care, 30, 203-209.

Nakawatase, Y., Taru, C., Tsutou, A., Shiotani, H., Kido, Y., Ohara, T., Ogawa, W. \& Miyawaki, I. (2007). Development of an evaluation scale for self-management behavior related to physical activity of type 2 diabetic patients. Diabetes Care, 30, 2843-2848.

Sarkar, U., Fisher, L. \& Schillinger, D. (2006). Is self-efficacy associated with diabetes selfmanagement across race/ethnicity and health literacy? Diabetes Care, 29, 823-829.

Stys, A.M. \& Kulkarni, K. (2007). Identification of self-care behaviours and adoption of lifestyle changes result in sustained glucose control and reduction of co-morbidities in type 2 diabetes. Diabetes Spectrum, 20(1), 55.

Thomas, N., Alder, E. \& Leese, G.P. (2005). Barriers to physical activity in patients with diabetes. Postgraduate Medical Journal, 80, 287-291.

Tumusiime, D.K. \& Frantz, J.M. (2006). Influence of previous participation in physical activity on its perceptions among tertiary institution students. African Journal for Physical, Health Education, Recreation and Dance, 12(3), 287-297.

World Health Organization .(2008). Chronic diseases. Available at: http://www.who.int/topics/chronic_diseases/en/ Retrieved September 4, 2008.

World Health Organization .(2006a). Chronic diseases in low and middle income countries. Available at http://www.who.int/chp/chronic_disease_report/ media.factsheet3pdf. Retrieved September 2, 2008.

World Health Organization .(2006b). Global strategy on healthy eating, physical activity and health - Implementing plan for the Latin America and the Caribbean (2006 - 2007). Available at: http://www.who.int/gh/ebwha/pdf_files/WHA59-REC3/WHA59_REC3-en.pdf.Retrieved September 8, 2008.

Zimmet, P., Alberti, K.G. \& Shaw, J. (2001). Global and societal implications of the diabetes epidemic. Nature, 414, 782-787. 